\title{
Conformational Switching Induced by Near-Infrared Laser Irradiation
}

\author{
Archna Sharma, Igor Reva, ${ }^{*}$ and Rui Fausto \\ Department of Chemistry, University of Coimbra, P-3004-535 Coimbra, Portugal
}

Received April 22, 2009; E-mail: reva@qui.uc.pt

Hydroxyacetone (HA) is an important starting material in the organic synthesis of gem-diols, acetals, and ketals. ${ }^{1}$ If such molecules are synthesized in interstellar medium, they may lead to the production of complex prebiotic chiral molecules, such as hydroxy aldehydes (sugars) and hydroxy acids. ${ }^{2}$ HA is formed in the atmosphere during oxidation of the important biogenic hydrocarbon, isoprene. ${ }^{3}$ Atmospheric $\mathrm{HA}$ reacts with an $\mathrm{OH}$ radical under sunlight irradiation ${ }^{4}$ and also contributes to the formation of secondary organic aerosols. ${ }^{5}$ The overall rate coefficient for the tropospherically relevant reaction, $\mathrm{HA}+\mathrm{OH}$, was proposed ${ }^{6}$ to depend on the initial conformation of the HA molecule.

Theoretical calculations on HA monomers in the gas phase $e^{7,8}$ show that there are only two conformers ( $\mathrm{Cc}$ and $\mathrm{Tt}$ ) of this compound (Figure 1). The lowest energy conformer of HA is Cc, whereas $\mathrm{Tt}$ was predicted to be higher in energy by $994 \mathrm{~cm}^{-1}$. Hence, the Cc conformer should represent $99 \%$ of the conformational equilibrium at room temperature, ${ }^{8}$ which is why only the $\mathrm{Cc}$ conformer has been observed so far. ${ }^{2,8,9}$

In this work, we report the first experimental observation of the elusive high-energy $\mathrm{Tt}$ conformer of hydroxyacetone. We show that, for HA isolated in a low-temperature Ar matrix, the Tt conformer can be produced by narrow-band near-infrared (NIR) vibrational excitation of the conformational ground state Cc. When isolated in an argon matrix at $15 \mathrm{~K}$, the photogenerated $\mathrm{Tt}$ form is stable. In the present study, the NIR-induced back transformation of the $\mathrm{Tt}$ conformer into the $\mathrm{Cc}$ form was also observed. This photoreversibility makes the HA molecule a system satisfying the criterion of a molecular switch. ${ }^{10}$

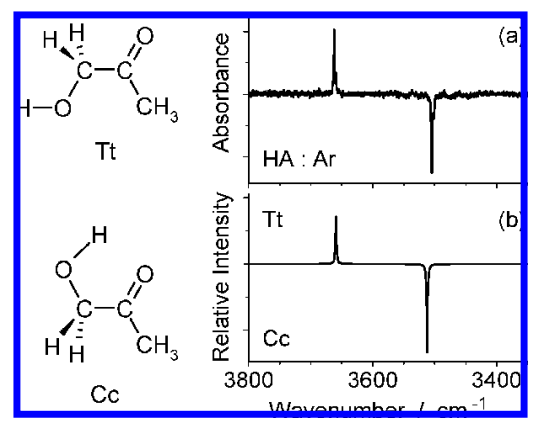

Figure 1. Left: Structures of Cc and Tt conformers of HA. Right: (a) The $\nu \mathrm{OH}$ region of the experimental infrared difference spectrum of HA monomers isolated in Ar matrix at $15 \mathrm{~K}$ (the spectrum after irradiation with $\lambda=6785 \mathrm{~cm}^{-1}$ minus the spectrum of the freshly deposited matrix). (b) Spectrum simulated with Lorentzian functions ( $\mathrm{fwhm}=2 \mathrm{~cm}^{-1}$ ). The calculated DFT(B3LYP)/6-311++G(d,p) frequencies in this region were scaled by a factor of 0.950 obtained by the least-squares linear fit. The difference spectrum was obtained as "Tt minus Cc".

In the experiments, tunable NIR radiation was provided by a Quanta-Ray MOPO-SL optical parametric oscillator (fwhm $\sim 0.2$ $\mathrm{cm}^{-1}$, repetition rate $10 \mathrm{~Hz}$, pulse energy $\sim 3 \mathrm{~mJ}$ ) pumped with a pulsed Nd:YAG laser. HA (Aldrich, >90\%) was premixed with argon in a 1:1000 ratio and deposited onto a CsI window at $15 \mathrm{~K}$. The FTIR spectra were recorded with $0.5 \mathrm{~cm}^{-1}$ resolution, in the $4000-400 \mathrm{~cm}^{-1}$ range. Other details of the experimental setup and of calculations are described elsewhere. ${ }^{8}$

In a freshly deposited argon matrix the HA monomer only exists in Cc geometry, which is separated by a global barrier of 1417 $\mathrm{cm}^{-1}$ from the higher-energy Tt form. ${ }^{8}$ Narrow-band NIR irradiation of the matrix at $6785 \mathrm{~cm}^{-1}$ led to a decrease of bands in the IR spectrum of the $\mathrm{Cc}$ form. Simultaneously, a set of new bands appeared due to a photogenerated species. The observed changes in the IR spectrum of HA are shown in Figures 1a and 2a. Comparison of the experimental difference spectrum with those calculated for the two conformers of HA (Figures $1 b$ and $2 b$ ) reveals doubtlessly that the photogenerated species corresponds to the higher energy Tt conformer. The assignment of the nine most intense infrared $\mathrm{Tt}$ absorptions (along with $\mathrm{Cc}$ ) is shown in Table 1.

The observed shifts of the $v \mathrm{OH}$ and $v \mathrm{C}=\mathrm{O}$ modes to higher frequencies (hypsochromic shifts) result from disruption of the intramolecular hydrogen bond on forming Tt. Absence of this interaction in $\mathrm{Tt}$ is responsible for its lower stability with respect to $\mathrm{Cc}$. This also leads to different forms of normal vibrations in $\mathrm{Tt}$ and $\mathrm{Cc}$ (see Table 1). Such differences occur for almost all angular deformations. The detailed discussion of the vibrational spectrum of HA-Tt is beyond the scope of this communication.

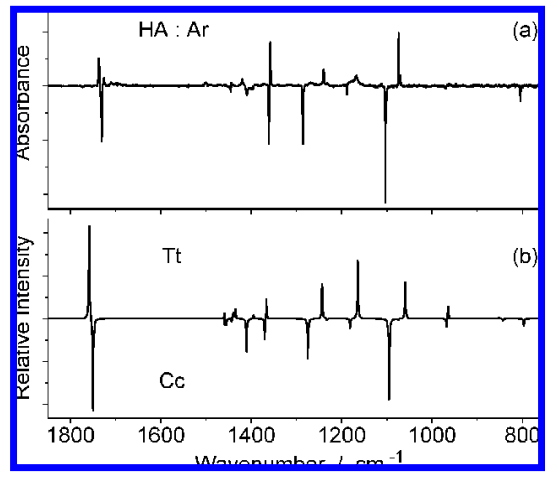

Figure 2. Fingerprint region of (a) the experimental spectrum of matrixisolated HA and (b) the simulated spectrum. The calculated frequencies in this region were scaled by a factor of 0.985 obtained by the least-squares linear fit. For other details see caption of Figure 1.

The experimental NIR absorption spectra of HA were not accessible in our experiments (nor in literature), and the choice of the irradiation conditions in the present study was not trivial. To locate the spectral position of the $\nu \mathrm{OH}$ overtone we resorted to anharmonic DFT calculations. The anharmonic $\mathrm{OH}$ stretching frequencies deviate from the experimental values by an order of magnitude less than those obtained in harmonic calculations. ${ }^{11}$ For the $\mathrm{Cc}$ conformer of $\mathrm{HA}$, we found the anharmonic $\nu \mathrm{OH}$ frequency $\left(3504 \mathrm{~cm}^{-1}\right.$ ) to differ by only $1 \mathrm{~cm}^{-1}$ from our experiment (Table 1 ). The first overtone $(2 \nu \mathrm{OH})$ of Cc conformer was predicted by our 
Table 1. Selected Experimental Frequencies and Infrared Intensities of Tt and Cc Conformers of Hydroxyacetone Isolated in an Argon Matrix at $15 \mathrm{~K}$ as Compared with the Values Calculated at the DFT(B3LYP)/6-311++G(d,p) Level

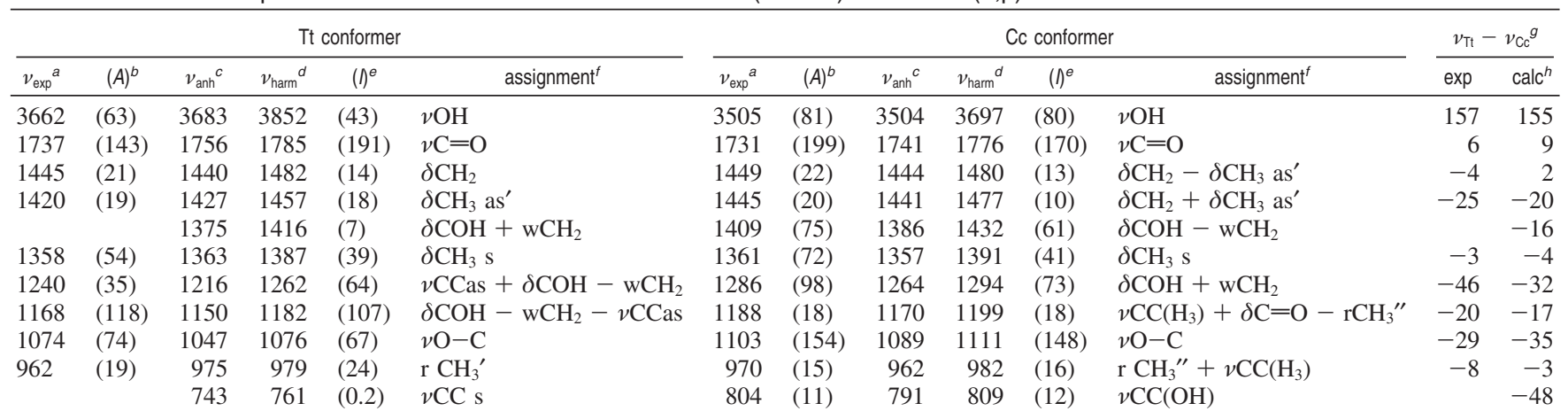

${ }^{a} v_{\text {exp }}$ - experimental frequency, $\mathrm{cm}^{-1} \cdot{ }^{b}(A)$ - relative experimental intensity. ${ }^{c} v_{\text {anh }}-$ calculated anharmonic frequency, $\mathrm{cm}^{-1}$. ${ }^{d} v_{\text {harm }}-\mathrm{calculated}$ harmonic frequency, $\mathrm{cm}^{-1}$. ${ }^{e}(I)$ calculated harmonic intensity, $\mathrm{km} \mathrm{mol}{ }^{-1} .{ }^{f}$ Based on PED calculation; $v$ - stretching, as - antisymmetric, $\mathrm{s}-\mathrm{symmetric}$, $\delta$ - bending, w - wagging, r - rocking, "plus" and "minus" designate "in-phase" and "in-antiphase" coupling. ${ }^{g}$ Frequency shift upon conformational change. ${ }^{h}$ Harmonic.

calculations at $6799 \mathrm{~cm}^{-1}$. A series of irradiations of matrix-isolated HA was carried out with NIR light of wavenumbers close to the predicted value $6799 \mathrm{~cm}^{-1}$. After an incremental search with a step of $3 \mathrm{~cm}^{-1}$, the irradiation at $6785 \mathrm{~cm}^{-1}$ was found to induce the $\mathrm{Cc} \rightarrow \mathrm{Tt}$ isomerization. Anharmonic calculations were also used to locate the frequency $\left(7188 \mathrm{~cm}^{-1}\right)$ of the $v \mathrm{OH}$ overtone in Tt. When the $\mathrm{Tt}$ conformer was generated in the matrix, the reverse $\mathrm{Tt} \rightarrow \mathrm{Cc}$ transformation could be successfully induced by NIR irradiation at $7138 \mathrm{~cm}^{-1}$.

The barrier for the $\mathrm{Tt} \rightarrow \mathrm{Cc}$ transformation was calculated to be $423 \mathrm{~cm}^{-1}\left(5 \mathrm{~kJ} \mathrm{~mol}^{-1}\right)$ only, ${ }^{8}$ yet HA-Tt was found to be stable in an Ar matrix at $15 \mathrm{~K}$. To the best of our knowledge, the observation of a long-lived reaction product $(\mathrm{Tt})$ generated in a low-temperature matrix by NIR excitation of $\nu \mathrm{OH}$ overtone is unprecedented. Previously, for small carboxylic acids ${ }^{12}$ isolated in low temperature matrices, narrow-band excitation of the $\nu \mathrm{OH}$ overtone was shown to promote generation of high-energy conformers. In the absence of NIR pumping, the photoproduced higher energy forms converted back to their low energy counterparts on a time scale of minutes. ${ }^{12}$ For carboxylic acids, this conversion only involves rotation of $\mathrm{OH}$ in the $\mathrm{O}=\mathrm{COH}$ fragment. In fact, only the hydrogen atom significantly changes its position by such rotation. It is the tunneling of this light particle that allows the cis-trans transformation in carboxylic acids to occur in darkness, ${ }^{13}$ although the barriers for such processes are greater than $30 \mathrm{~kJ} \mathrm{~mol}^{-1}$.

For the HA molecule, however, the isomerization between $\mathrm{Cc}$ and $\mathrm{Tt}$ forms involves reorganization of the heavy atom backbone. The heavy oxygen atom significantly changes its position in the $\mathrm{Tt} \rightarrow \mathrm{Cc}$ transformation. Tunneling of atoms as heavy as oxygen should be unlikely: with other heavy elements, there are mainly reports on tunneling of carbon atoms. ${ }^{14}$ Therefore, the $\mathrm{Tt} \rightarrow \mathrm{Cc}$ conversion does not occur for HA at $15 \mathrm{~K}$, although the barrier for this process is as low as $5 \mathrm{~kJ} \mathrm{~mol}^{-1}$. However, if thermally activated, this relaxation may occur over the barrier. ${ }^{15}$ Indeed, the $\mathrm{Tt} \rightarrow \mathrm{Cc}$ transformation was induced by annealing of the sample to a temperature of $28 \mathrm{~K}$ ! The rearrangement of the argon atoms in this thermal back conversion process should be nonsignificant, since the changes of the baseline in the experimental spectra observed upon annealing were negligible.

In conclusion, we report the unusual observation of conformational change, involving rotation of molecular moieties with heavy atoms, induced by absorption of a NIR photon. The Tt conformer of HA was produced in solid argon by excitation of the $\nu \mathrm{OH}$ overtone of the $\mathrm{Cc}$ form. The experimental evidence for generation of the $\mathrm{Tt}$ form is supported by theoretical vibrational analysis. We hope that the present experimental findings will stimulate further theoretical and experimental studies on the overtone-induced chemical transformations, ${ }^{16}$ particularly those concerning vibrational energy transfer and redistribution due to anharmonic couplings in polyatomic molecules.

Acknowledgment. This work was supported by the Portuguese Science Foundation (FCT) Grant No. SFRH/BPD/ 31840/2006 and Research Project PTDC/QUI/71203/2006. We are grateful to Dr. Leszek Lapinski, Dr. Hugh Burrows, and Dr. Luis Arnaut for the helpful discussions.

\section{References}

(1) Carey, F. A. Organic Chemistry, 7th ed.; McGraw-Hill: 2007.

(2) Apponi, A. J.; Hoy, J. J.; Halfen, D. T.; Ziurys, L. M.; Brewster, M. A. Astrophys. J. 2006, 652, 1787-1795.

(3) Dillon, T. J.; Horowitz, A.; Hölscher, D.; Crowley, J. N.; Vereecken, L.; Peeters, J. Phvs. Chem. Chem. Phvs. 2006, 8, 236-246.

(4) Grosjean, D.; Williams, E. L. II.; Grosjean, E. Environ. Sci. Technol. 1993, $27,830-840$.

(5) Lee, A.; Goldstein, A. H.; Kroll, J. H.; Ng, N. L.; Varutbangkul, V.; Flagan, R. C.; Seinfeld, J. H. J. Geophys. Res. Atm. 2006, 111, D17305.

(6) Galano, A. J. Phys. Chem. A 2006, 110, 9153-9160.

(7) Jalbout, A. F.; Contreras-Torres, F. F.; Adamowicz, L. Int. J. Quantum Chem. 2008, 108, 279-288.

(8) Sharma, A.; Reva, I.; Fausto, R. J. Phvs. Chem. A 2008, 112, 5935-5946.

(9) Kattija-Ari, M.; Harmony, M. D. Int. J. Quantum Chem. - Quant. Chem. Symp. 1980, 17, 443-453.

(10) Feringa, B. L., Ed. Molecular Switches: Wiley: 2001.

(11) (a) Akai, N.; Katsumoto, Y.; Ohno, K.; Aida, M. Chem. Phvs. Lett. 2005, 413, 367-372. (b) Adesokan, A. A.; Gerber, R. B. J.Phvs. Chem. A 2009, $113,1905-1912$

(12) (a) Pettersson, M.; Lundell, J.; Khriachtchev, L.; Räsänen, M. J. Am. Chem. Soc. 1997, 119, 11715-11716. (b) Maçôas, E. M. S.; Khriachtchev, L.; 16188-16189.

(13) Khriachtchev, L. J. Mol. Struct. 2008, 880, 14-22.

(14) (a) For example: Datta, A.; Hrovat, D. A.; Borden, W. T. J. Am. Chem. Soc. 2008, 130, 6684-6685. (b) Berger, R. Angew. Chem., Int. Ed. 2004, 43, 398-401. (c) Zuev, P. S.; Sheridan, R. S.; Albu, T. V.; Truhlar, D. G.; Hrovat, D. A.; Borden, W. T. Science 2003, 299, 867-870. (d) Redington, R. L. J. Chem. Phys. 1998, 109, 10781.

(15) Reva, I. D.; Stepanian, S. G.; Adamowicz, L.; Fausto, R. Chem. Phys. Lett. 2003, 374, 631-638.

(16) Feature Article: Vaida, V. J. Phys. Chem. A 2009, 113, 5-18.

JA903211F 